\title{
Correlation of four potential biomarkers of liver fibrosis with liver function and grade of hepatic fibrosis in a neonatal cholestatic rat model
}

\author{
NING TANG, YAPING ZHANG, ZEYU LIU, XUEMEI AI and QINGHONG LIANG \\ Department of Pediatrics, Third Hospital of Hebei Medical University, Shijiazhuang, Hebei 050051, P.R. China
}

Received April 18, 2016; Accepted March 17, 2017

DOI: $10.3892 / \mathrm{mmr} .2017 .6588$

\begin{abstract}
The present study investigated the correlation between four serum biomarkers of liver fibrosis, liver function and pathological hepatic fibrosis grade in neonatal cholestatic rats. A total of 38 Sprague-Dawley rats, aged 3 weeks, were randomly assigned to the experimental group (EG), control group (CG) and the blank control group (BCG). EG received intragastric administration of $1 \% \alpha$-naphthylisothiocyanate, $75 \mathrm{mg} / \mathrm{kg}$, to induce acute cholestasis liver injury, CG and BCG were set as control groups. Blood samples from all groups were collected $48 \mathrm{~h}$ following the procedure. The levels of liver function markers, and four biomarkers of liver fibrosis in serum, were measured and sections of liver tissue were stained for pathological analysis. The results of the present study demonstrated that the degree of hepatic fibrosis in EG, in the serum levels or by pathological analysis, was markedly more evident compared with the CG. Several indices of four biomarkers for liver fibrosis in serum were identified and correlated with the levels of liver function markers. The pathological hepatic fibrosis grade was correlated with $\gamma$-glutamyl transferase $(\gamma$-GT) and Hyaluronic acid (HA). Therefore, HA and $\gamma$-GT were positively correlated with the grade of hepatic fibrosis, indicating their efficacy as biomarkers of infantile cholestatic hepatic fibrosis.
\end{abstract}

\section{Introduction}

Arising from either a functional defect in bile formation at the hepatocyte or from impaired secretion and flow capability of the bile duct, cholestasis is the primary cause of hospitalization in children with liver diseases (1). In the rat model of cholestasis, bile duct epithelial cells are synchronized with

Correspondence to: Dr Yaping Zhang, Department of Pediatrics, Third Hospital of Hebei Medical University, 139 Ziqiang Road, Shijiazhuang, Hebei 050051, P.R. China

E-mail: yapingzhang@aliyun.com

Key words: neonatal cholestatic rat, liver function markers, four biomarkers of liver fibrosis, hepatic fibrosis grade in pathology, correlation analysis hepatic stellate cells with respect to activation and proliferation, inducing extracellular matrix deposition and hepatic fibrosis development $(2,3)$. Pathological examination of liver tissue is the primary method of diagnosing hepatic fibrosis; however, due to its invasiveness, the use of it is significantly limited in infants. Therefore, developing a non-invasive method for detecting hepatic fibrosis may provide an important clinical advancement.

Known to cause acute cholestasis, $\alpha$-naphthylisothiocyanate (ANIT), a highly toxic substance (4), has been used to induce swelling and necrosis of the bile ducts, resulting in hyperplasia and an inflammatory reaction around the ductus biliferi interlobulares. ANIT obstructs the bile ducts causing cholestasis, hepatic parenchymal cell damage and hepatic fibrosis, and has been identified to be a useful agent for studying cholestasis. In the present study, ANIT was used to induce cholestatic hepatic injury in neonatal rats in order to analyze four suspected biomarkers of the disease. The present study was designed to assess the correlation between the four biomarkers, and enzymes indicative of liver function and hepatic fibrosis. An effective, simple, and non-invasive way of diagnosing hepatic fibrosis in clinical settings may become available in the future.

\section{Materials and methods}

Rodent model and rearing conditions. A total of 38 healthy Sprague-Dawley rats (weight, 50-70 g; age, 3 weeks; male, 19; female, 19) were obtained from the Laboratory Animal Center of Hebei Medical University (Shijiazhuang, China). Animals were kept at an ambient temperature of $25 \pm 2^{\circ} \mathrm{C}$, with a $12: 12 \mathrm{~h}$ light/dark cycle, and were given free access to standard laboratory chow and tap water. All rats were allowed to acclimate for $24 \mathrm{~h}$ prior to experimentation. All procedures involving animals were reviewed and approved by the Institutional Animal Care and Use Committee of Hebei Medical University. The animal protocol was designed to minimize pain and discomfort to the animals.

Group designations and treatment. Animals were assigned at random to one of three groups: The experimental model group (EG); the vehicle control group (CG); and the blank control group (BCG). The EG rats were administered $1 \%$ ANIT dissolved in corn oil (75 mg/kg; Sigma-Aldrich; Merck KGaA, Darmstadt, Germany) intragastrically, in order to induce 
disease development. Rats in the CG were treated with an equivalent volume of corn oil compared with the total volume administered to the EG rats; the BCG rats were untreated, and fasted for $12 \mathrm{~h}$ prior to and following treatment. Treatments were administered daily for 2 days until sacrifice and all procedures were conducted with approval of the Council on Animal Care of Academia Sinica (Hebei Medical University, Shijiazhuang, China).

Sample collection and preparation. Following $48 \mathrm{~h}$ of treatment, all animals were sacrificed, and blood and liver samples were collected. Blood samples from the heart were evaluated for markers indicative of liver function and liver fibrosis, while liver samples were preserved for histological and immunohistochemical examination in $10 \%(\mathrm{w} / \mathrm{v})$ neutral formaldehyde. Histological surgery and evaluation were performed by a certified veterinary pathologist.

Detection of biomarkers of hepatic fibrosis. Radioimmunoassays from Beijing North Institute of Biotechnology (Beijing, China) were used in conjunction with a $\gamma$-radioimmunoassay counter (XH-6020; Xi'an Nuclear Instrument Factory, Shaanxi, China) in accordance with the manufacturers' protocol. Rats fasted overnight and had 2-3 $\mathrm{ml}$ of venous blood collected in pro-coagulant tubes, which were centrifuged at $1,006 \mathrm{x} \mathrm{g}$ for $3 \mathrm{~min}$ to isolate the serum. All serum samples were stored at $-20^{\circ} \mathrm{C}$, and measurements of hyaluronic acid (HA), procollagen type III (PCIII), laminin (LN) and collagen type IV (cIV) were taken.

Measurement of liver function in the serum. Detection kits specific for liver function biomarkers [alanine aminotransferase (ALT); aspartate aminotransferase (AST); total bilirubin (TBIL); direct bilirubin (DBIL); indirect bilirubin (IBIL); $\gamma$-glutamyl transferase $(\gamma$-GT); cholinesterase (CHE); and total bile acids (TBA)] were purchased from Johnson \& Johnson (New Brunswick, NJ, USA) and used in conjunction with an automatic biochemical analyzer (AU5400TM; Olympus Corporation, Tokyo, Japan). Blood samples were collected from each mouse and processed as previously described (5). Measurements of liver function biomarkers were based on assays using the initial rate method (5), and measurements of TBIL, DBIL and IBIL were based on colorimetric assays.

Hematoxylin and eosin staining. Formaldehyde-fixed paraffin embedded (FFPE) liver specimens were processed into 4- $\mu$ m-thick sections mounted onto glass slides, stained with hematoxylin and eosin and examined with light microscopy (6). This procedure was performed by a certified veterinary pathologist. Digital images were quantitatively and semi-quantitatively graded for hepatic fibrosis utilizing the scoring criteria established by Farleigh et al (7). Score 0 , no fibrosis and no hyperplasia of collagen in liver; score 1, few collagen fibrils extended from the central vein to the portal tract $(<25 \%)$; score 2, apparent collagen fibril extension without encompassing the whole lobule (25-50\%); score 3 , collagen fibrils extended into and encompassing the whole lobule (50-75\%); and score 4, diffuse extension of collagen fibrils and formation of pseudo-lobule $(>75 \%)$.
Masson staining. This procedure was performed by a certified veterinary pathologist. FFPE liver samples of $6 \mu \mathrm{m}$ thickness were mounted onto glass slides and stained with masson (Shanghai, China) (8).

Picric acid-sirius red staining. This procedure was performed by a certified veterinary pathologist. FFPE liver samples were sliced into $6-\mu \mathrm{m}$-thick sections, mounted onto glass slides, and stained with picric acid-sirius red $(0.1 \%$ sirius red in saturated aqueous picric acid for 10-15 $\mathrm{min}$ at room temperature of $20-25^{\circ} \mathrm{C}$ ) to detect hepatic fibrosis. Stained sections were examined using a polarizing microscope (BX53-p; Olympus Corporation; magnification, $\mathrm{x} 400$ ).

Statistical analysis. Statistical analysis was performed using SPSS software (version 19.0; IBM SPSS, Armonk, NY, USA) and the results are expressed as the mean \pm the standard deviation. A Student's t-test was used for normally distributed data, while the Wilcoxon rank sum test was used for non-normally distributed data; additionally, the rank sum test was used for disease grade data. Partial correlation analysis was used to evaluate the association between each liver function indicator and the four biomarkers. Data from the pathological grading of tissue samples were analyzed using Spearman's rank correlation coefficient. $\mathrm{P}<0.05$ was considered to indicate a statistically significant difference.

\section{Results}

Serum levels of enzymes indicative of liver function were elevated in the EG. Serum levels of ALT, AST, TBIL, DBIL, IBIL, $\gamma$-GT and TBA in neonatal rats with cholestasis were significantly increased, compared with rats in the CG and BCG $(\mathrm{P}<0.01$; Table I). CHE levels in neonatal rats with cholestasis were unaltered compared with the control groups.

Serum levels of $H A, L N$, and cIV were significantly elevated in the EG. Among the four biomarkers of hepatic fibrosis, serum levels of HA, LN and cIV in neonatal rats with cholestasis were significantly increased compared with the control groups $(\mathrm{P}<0.01$; Table II). PCIII levels in neonatal rats with cholestasis were unaltered compared with the control groups.

An increased grade of hepatic fibrosis was associated with animals in the EG. The grade of pathological hepatic fibrosis was notably increased in the cholestasis group compared with the control groups $(\mathrm{P}<0.01$; Table III).

\section{Hepatic tissue pathological staining}

Hematoxylin and eosin staining. Hepatic lobules in the CG and BCG were holonomic and clear, and the cells were arranged in a funicular form with no infiltration in the portal area. Samples from the EG exhibited fatty degeneration, edema, necrosis and apoptosis in $25-75 \%$ of each sample, with damaged or absent hepatic lobules. Cholestasis was observed in a majority of the hepatocytes and bile ducts, with few normal hepatocytes. There was apparent fibrosis and necrosis in a majority of the 
Table I. Variation of liver function test in neonatal cholestatic rats.

\begin{tabular}{|c|c|c|c|}
\hline \multirow[b]{2}{*}{ Factor } & \multicolumn{3}{|c|}{ Group } \\
\hline & $\begin{array}{l}\text { Vehicle control } \\
\qquad(\mathrm{n}=8)\end{array}$ & $\begin{array}{l}\text { Negative control } \\
\qquad(\mathrm{n}=7)\end{array}$ & $\begin{array}{l}\text { Cholestasis } \\
\quad(n=18)\end{array}$ \\
\hline ALT, U/1 & $48.88 \pm 14.04$ & $36.14 \pm 11.34$ & $633.17 \pm 248.80^{\mathrm{a}, \mathrm{b}}$ \\
\hline AST, U/l & $349.50 \pm 114.41$ & $320.71 \pm 148.83$ & $2080.17 \pm 704.39^{\mathrm{a}, \mathrm{b}}$ \\
\hline TBIL, $\mu \mathrm{mol} / 1$ & $3.28 \pm 0.87$ & $2.43 \pm 0.67$ & $144.81 \pm 24.90^{\mathrm{a}, \mathrm{b}}$ \\
\hline $\mathrm{DBIL}, \mu \mathrm{mol} / \mathrm{l}$ & $2.85 \pm 0.91$ & $1.93 \pm 0.69$ & $132.79 \pm 23.43^{\mathrm{a}, \mathrm{b}}$ \\
\hline IBIL, $\mu \mathrm{mol} / \mathrm{l}$ & $0.43 \pm 0.29$ & $0.50 \pm 0.33$ & $12.02 \pm 5.60^{\mathrm{a}, \mathrm{b}}$ \\
\hline$\gamma-\mathrm{GT}, \mathrm{U} / 1$ & $0.75 \pm 1.04$ & $0.14 \pm 1.57$ & $19.94 \pm 10.37^{\mathrm{a}, \mathrm{b}}$ \\
\hline CHE, KU/1 & $0.26 \pm 0.04$ & $0.27 \pm 0.03$ & $0.26 \pm 0.04$ \\
\hline TBA, $\mu \mathrm{mol} / 1$ & $198.01 \pm 120.47$ & $113.03 \pm 87.37$ & $675.66 \pm 84.74^{a, b}$ \\
\hline
\end{tabular}

Data are expressed as the mean \pm standard deviation. ${ }^{a} \mathrm{P}<0.01$ vs. vehicle control; ${ }^{\mathrm{b}} \mathrm{P}<0.01 \mathrm{vs}$. negative control. ALT, alanine aminotransferase; $\gamma$-GT, $\gamma$-glutamyl transferase; TBIL, total bilirubin; DBIL, direct bilirubin; TBA, total bile acids; CHE, cholinesterase; AST, aspartate aminotransferase; IBIL, indirect bilirubin.

Table II. Variation of degrees of hepatic fibrosis in neonatal cholestatic rats.

\begin{tabular}{lccc}
\hline & \multicolumn{2}{c}{ Group } & $\begin{array}{c}\text { Cholestasis } \\
(\mathrm{n}=18)\end{array}$ \\
\cline { 2 - 4 } Factor & $\begin{array}{c}\text { Vehicle control } \\
(\mathrm{n}=7)\end{array}$ & $\begin{array}{c}\text { Negative control } \\
(\mathrm{n}=6)\end{array}$ & $2004.18 \pm 479.86^{\mathrm{a}, \mathrm{b}}$ \\
$\mathrm{HA}, \mathrm{ng} / \mathrm{ml}$ & $1030.07 \pm 541.19$ & $1018.95 \pm 555.13$ & $45.52 \pm 2.16$ \\
$\mathrm{PCIII}, \mathrm{ng} / \mathrm{ml}$ & $46.63 \pm 2.63$ & $36.20 \pm 9.65$ & $48.51 \pm 6.51^{\mathrm{a}, \mathrm{b}}$ \\
$\mathrm{LN}, \mathrm{ng} / \mathrm{ml}$ & $34.41 \pm 10.81$ & $27.57 \pm 6.25$ & $30.28 \pm 10.82^{\mathrm{a}, \mathrm{b}}$ \\
$\mathrm{cIV}, \mathrm{ng} / \mathrm{ml}$ & $20.08 \pm 2.70$ & $18.33 \pm 5.59$ & 3 \\
\hline
\end{tabular}

Data are expressed as the mean \pm standard deviation. ${ }^{\mathrm{a}} \mathrm{P}<0.01$ vs. vehicle control; ${ }^{\mathrm{b}} \mathrm{P}<0.01 \mathrm{vs}$. negative control. HA, hyaluronic acid; $\mathrm{PCIII}$, procollagen type III; LN, laminin; cIV, collagen type IV.

Table III. Variation of pathological hepatic fibrosis grade in neonatal cholestatic rats.

\begin{tabular}{lccc}
\hline & \multicolumn{3}{c}{ Group } \\
\cline { 2 - 4 } & $\begin{array}{l}\text { Vehicle } \\
\text { control } \\
(\mathrm{n}=10)\end{array}$ & $\begin{array}{c}\text { Negative } \\
\text { control } \\
(\mathrm{n}=8)\end{array}$ & $\begin{array}{c}\text { Cholestasis } \\
(\mathrm{n}=20)\end{array}$ \\
\hline $\begin{array}{l}\text { Factor } \\
\text { grade }\end{array}$ & $0.44 \pm 0.53$ & $0.13 \pm 0.35$ & $2.05 \pm 0.62^{\mathrm{a}, \mathrm{b}}$ \\
\hline
\end{tabular}

Data are expressed as the mean \pm standard deviation. ${ }^{\mathrm{a}} \mathrm{P}<0.01$ vs. vehicle control; ${ }^{b} \mathrm{P}<0.01$ vs. negative control.

portal areas accompanied by severe infiltration of neutrophilic granulocytes and eosinophilic granulocytes. Additionally, a fibrous septum divided necrotic hepatic lobules into pseudolobules (Fig. 1A-D).
Masson staining. There was a buildup of collagen in the hepatic tissue of samples from the EG which was not observed in the control groups (Fig. 1E).

Sirius red-saturated picric acid staining. Hepatic lobules of the experimental group were observed to exhibit a large population of collagen fibers compared with the control groups. Among the fibers in the EG, thick yellow and red fibers had greater refractivity and appeared as circles around the central vein and the clearance of hepatic sinus, star-shaped around the portal area, or stretched into the lobule forming interlobular separations. A few slender green fibers were observed to be distributed in portal area, small blood vessels, and central vein, and additionally were observed to be discontinuously distributed around the clearance of the hepatic sinus (Fig. 1F).

Correlation analyses between liver function tests and the four biomarkers of hepatic fibrosis in neonatal rats with cholestasis. HA was positively correlated with ALT and $\gamma-\mathrm{G}$ $(\mathrm{r}=0.47, \mathrm{P}<0.05 ; \mathrm{r}=0.53, \mathrm{P}<0.05$, respectively; Fig. $2 \mathrm{~A}$ and $\mathrm{B})$, 

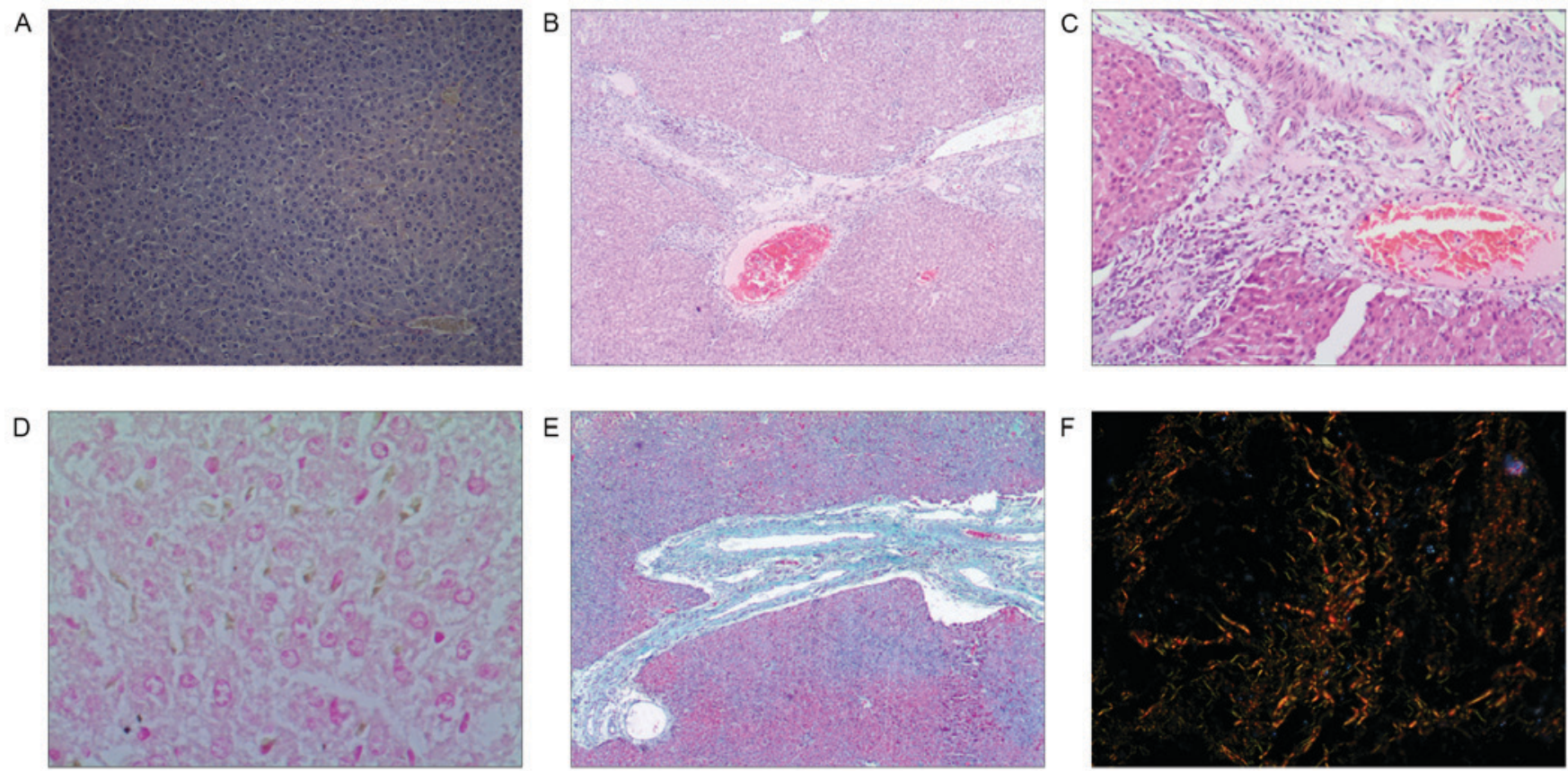

Figure 1. Results of hepatic tissue pathological staining. (A) Normal hepatic lobules stained with hematoxylin and eosin. Magnification, x200. Hepatic lobules were observed to be holonomic and clear. Hepatic cells were arranged in funicular form. Almost no proliferation of fiber tissue was observed. (B) Liver of neonatal cholestatic rat stained with hematoxylin and eosin. Magnification, x40. Fibrosis was observed, and the fibrous septum divided necrotic hepatic lobules into pseudolobules. (C) Liver of neonatal cholestatic rat stained with hematoxylin and eosin. Magnification, x100. Fibrosis with severe neutrophilic and eosinophilic granulocyte infiltration was observed. (D) Liver of neonatal cholestatic rat stained with hematoxylin and eosin. Magnification, $\mathrm{x} 400$. Cholestasis was observed in hepatocytes and bile ducts. (E) Liver of neonatal cholestatic rat stained with masson. Magnification, $x 40$. A large number of green collagen fibers proliferated around blood vessels and bile ducts. (F) Liver of neonatal cholestatic rat stained with sirius red-saturated picric acid. Magnification, x400. Large, thick yellow and red fibers with strong refractivity and few slender green fibers with weak birefringence were observed.

while cIV was only observed to positively correlate with $\gamma$-GT ( $r=0.50$, P<0.05; Fig. 2C; Table IV). Additionally, PCIII was positively correlated with TBIL, DBIL, $\gamma$-GT and TBA $(\mathrm{r}=0.65, \mathrm{P}<0.01 ; \mathrm{r}=0.65, \mathrm{P}<0.01 ; \mathrm{r}=0.54, \mathrm{P}<0.05 ; \mathrm{r}=0.62$, $\mathrm{P}<0.01$, respectively; Fig. 2D-G).

Correlation analysis of liver function and hepatic fibrosis grade. Pathological hepatic fibrosis grade was positively correlated with $\gamma$-GT ( $r=0.62, \mathrm{P}<0.01$; Fig. $2 \mathrm{H}$; Table V) among the biomarkers of liver function of the experimental group.

Correlation analysis of serum biomarkers of liver fibrosis and hepatic fibrosis grade. Pathological hepatic fibrosis grade was positively correlated with $\mathrm{HA}(\mathrm{r}=0.83, \mathrm{P}<0.01$; Fig. 2; Table VI) in the four biomarkers of hepatic fibrosis of the experimental group.

\section{Discussion}

Non-invasive testing of liver fibrosis is among the fastest evolving areas in the study of liver diseases (9). Liver biopsy has been the standard method for the screening and surveillance of liver fibrosis. However, this procedure is associated with certain limitations. Liver biopsies are only able to sample a small portion of the liver and, therefore, sampling errors may occur, particularly when smaller-sized biopsies are analyzed. Histopathological diagnosis may be subject to both intraand inter-observer variability, even with validated scoring systems (10). Liver biopsy is an invasive procedure with associated adverse events, including pain, which occurs in $20 \%$ of patients, and major complications, including bleeding and liver laceration (11). Therefore, liver biopsy is a particularly risky procedure in neonates. Further research is required to identify non-invasive markers of liver fibrosis, particularly in neonatal liver disease, where prompt, safe and reliable diagnosis is required. In the present study, the utility of four possible markers of fibrosis was assessed: HA, PCIII, LN, cIV. Cholestatic jaundice and eventual fibrosis was induced using ANIT.

Following its identification 30 years ago as a chemical inducer of acute intrahepatic cholestasis, ANIT has been utilized in several animal studies of cholestatic liver disease (12). Although its mechanism of action remains to be completely elucidated, the early stages of liver injury induced by ANIT are characterized by increasing levels of AST, ALT and TBIL (13). As the damage progresses, epithelial cells in the bile ducts swell and undergo necrosis, leading to capillary bile duct hyperplasia and an inflammatory reaction which further obstructs the duct. The application of ANIT induces cholestasis, liver parenchymal cell damage and cholestatic jaundice. Previous studies have demonstrated that, in the process of liver injury caused by treatment with ANIT, serum levels of ALT, TBA and other markers increase until a peak is reached at $48 \mathrm{~h}(14,15)$.

The results of the present study demonstrated the successful generation of a model of acute cholestatic hepatic fibrosis, using hematoxylin and eosin, masson and sirius red-saturated picric acid staining. The histopathologic findings of fibrosis demonstrated the association of the four markers of fibrosis (HA, PCIII, LN, and cIV) with the onset of fibrosis. 

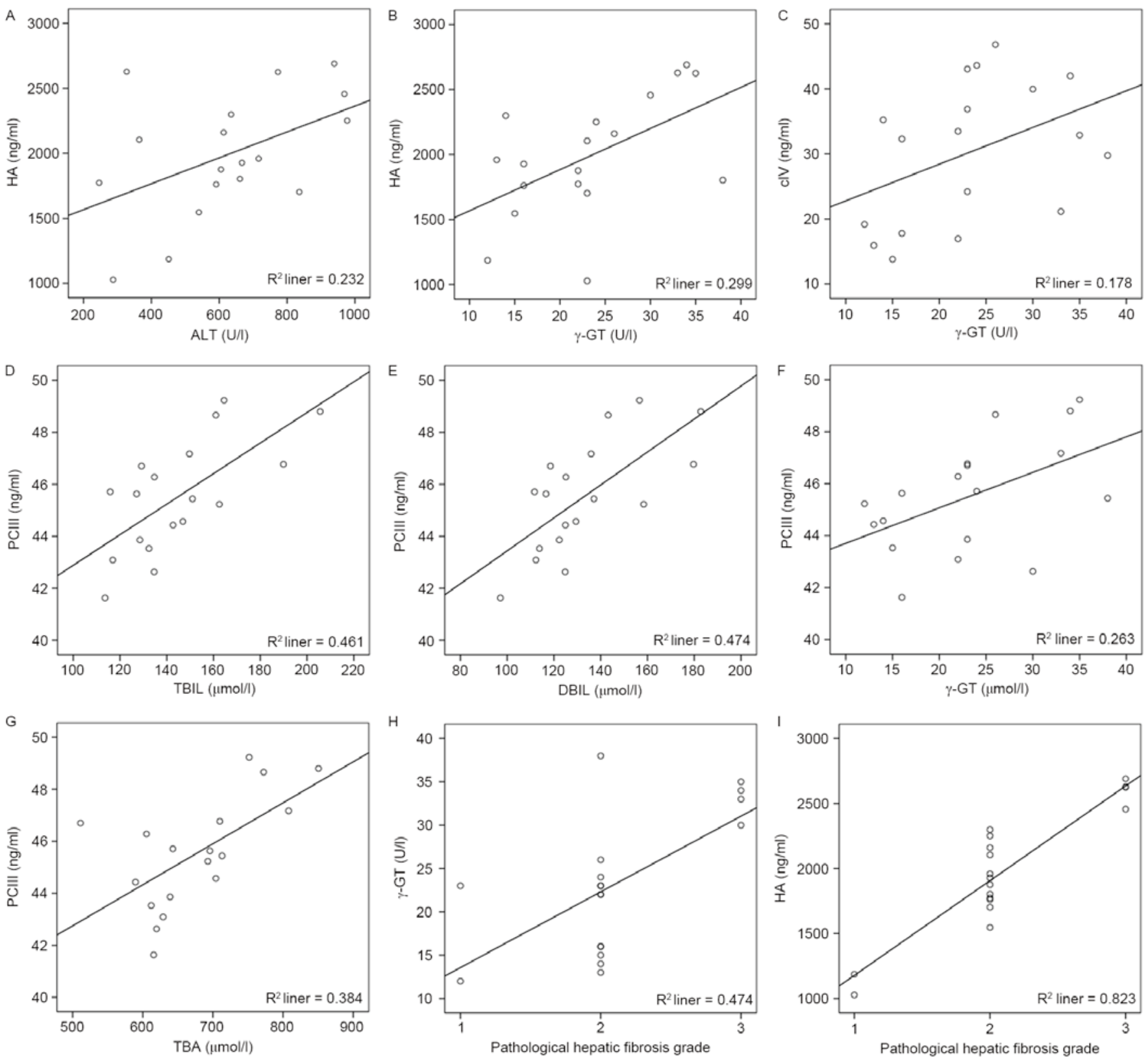

Figure 2. Correlation analyses in neonatal rats with cholestasis. (A) HA and ALT were positively correlated in neonatal cholestatic rats. $\mathrm{n}=18$; $\mathrm{r}=0.47$; $\mathrm{P}<0.05$. (B) HA and $\gamma$-GT were positively correlated in neonatal cholestatic rats. $\mathrm{n}=18 ; \mathrm{r}=0.53 ; \mathrm{P}<0.05$. (C) cIV and $\gamma$-GT were positively correlated in neonatal cholestatic rats. $\mathrm{n}=18 ; \mathrm{r}=0.50 ; \mathrm{P}<0.05$. (D) PCIII and TBIL were positively correlated in neonatal cholestatic rats. $\mathrm{n}=18$; $\mathrm{r}=0.65$; $\mathrm{P}<0.01$. (E) $\mathrm{PCIII}$ and $\mathrm{DBIL}$ were positively correlated in neonatal cholestatic rats. $\mathrm{n}=18 ; \mathrm{r}=0.65 ; \mathrm{P}<0.01$. (F) PCIII and $\gamma$-GT were positively correlated in neonatal cholestatic rats. $\mathrm{n}=18$; $\mathrm{r}=0.54$; $\mathrm{P}<0.05$. (G) PCIII and TBA were positively correlated in neonatal cholestatic rats. $\mathrm{n}=18 ; \mathrm{r}=0.616 ; \mathrm{P}<0.01$. (H) Pathological hepatic fibrosis grade and $\gamma$-GT were positively correlated in neonatal cholestatic rats. $\mathrm{n}=18 ; \mathrm{r}=0.62 ; \mathrm{P}<0.01$. (I) Pathological hepatic fibrosis grade and $\mathrm{HA}$ were positively correlated in neonatal cholestatic rats. $\mathrm{n}=18 ; \mathrm{r}=0.83$; $\mathrm{P}<0.01$. HA, hyaluronic acid; ALT, alanine aminotransferase; $\gamma$-GT, $\gamma$-glutamyl transferase; cIV, collagen type IV; PCIII, procollagen type III; TBIL, total bilirubin; DBIL, direct bilirubin; TBA, total bile acids.

HA is primarily absorbed and degraded by hepatic sinusoidal endothelial cells. Following injury, cytokines mediate the interaction of cells leading to the activation of hepatic sinusoidal endothelial cells and the production of HA has been observed (16). A previous report demonstrated that hepatic fibrosis develops as disease progresses, and a positive correlation has been identified between increases in serum HA and the severity of histological lesions (17). Additionally, this rise in serum HA may be used to accurately and sensitively interpret the damage status of hepatocytes as well as the fiber accumulation in the liver $(18,19)$. The results of the present study are consistent with previous observations that HA is a viable marker of fibrosis, particularly in children with metabolic liver injury. Levels of $\mathrm{HA} \geq 2,100 \mathrm{ng} / \mathrm{ml}$ were observed to be correlated with F2, F3 or F4 fibrosis (20).

An additional component of the hepatocyte basement membrane is cIV, the expression levels of which have been observed to increase in the early stages of hepatic fibrosis. It is during this phase of early fibrosis that cIV is deposited, prior to the onset of permanent liver damage (21). Therefore, early detection of cIV, as demonstrated in the present study, is a viable marker of liver fibrosis. In conjunction with cIV, 
Table IV. Correlation analysis of degrees of hepatic fibrosis and liver function test in neonatal cholestatic rats.

Factor

\begin{tabular}{|c|c|c|c|c|c|c|c|c|}
\hline \multirow[b]{2}{*}{ Factor } & \multicolumn{2}{|c|}{$\mathrm{HA}, \mathrm{ng} / \mathrm{ml}$} & \multicolumn{2}{|c|}{ PCIII, ng/ml } & \multicolumn{2}{|c|}{$\mathrm{LN}, \mathrm{ng} / \mathrm{ml}$} & \multicolumn{2}{|c|}{$\mathrm{cIV}, \mathrm{ng} / \mathrm{ml}$} \\
\hline & $\mathrm{r}$ & P-value & $\mathrm{r}$ & P-value & $\mathrm{r}$ & P-value & $\mathrm{r}$ & P-value \\
\hline ALT, U/1 & 0.47 & $<0.05$ & 0.27 & N.S & 0.07 & N.S & 0.34 & N.S \\
\hline AST, U/1 & 0.39 & N.S & 0.10 & N.S & 0.19 & N.S & 0.38 & N.S \\
\hline $\mathrm{TBIL}, \mu \mathrm{mol} / \mathrm{l}$ & 0.29 & N.S & 0.65 & $<0.01$ & -0.20 & N.S & 0.10 & N.S \\
\hline $\mathrm{DBIL}, \mu \mathrm{mol} / 1$ & 0.26 & N.S & 0.65 & $<0.01$ & -0.17 & N.S & 0.13 & N.S \\
\hline IBIL,$\mu \mathrm{mol} / 1$ & 0.26 & N.S & 0.08 & N.S & -0.15 & N.S & -0.04 & N.S \\
\hline$\gamma-\mathrm{GT}, \mathrm{U} / 1$ & 0.53 & $<0.05$ & 0.54 & $<0.05$ & 0.12 & N.S & 0.50 & $<0.05$ \\
\hline CHE, KU/1 & -0.27 & N.S & -0.17 & N.S & -0.01 & N.S & -0.10 & N.S \\
\hline $\mathrm{TBA}, \mu \mathrm{mol} / \mathrm{l}$ & 0.43 & N.S & 0.62 & $<0.01$ & -0.17 & N.S & 0.32 & N.S \\
\hline
\end{tabular}

N.S, not significant; HA, hyaluronic acid; ALT, alanine aminotransferase; $\gamma$-GT, $\gamma$-glutamyl transferase; cIV, collagen type IV; PCIII, procollagen type III; TBIL, total bilirubin; DBIL, direct bilirubin; TBA, total bile acids; LN, laminin; CHE, cholinesterase; AST, aspartate aminotransferase; IBIL, indirect bilirubin.

LN is an additional potential marker of liver fibrosis. Liver specimens obtained from patients with alcoholic liver disease and chronic viral hepatitis have been demonstrated to exhibit a strong reaction with antibodies in the peri-sinusoidal space, including staining for LN (21). However, few studies have investigated LN levels in neonatal cholestasis, leading to fibrosis.

ALT, which is predominantly located in the hepatic cytoplasm, is a sensitive indicator of damage to hepatocytes. An additional marker, increased serum levels of $\gamma-\mathrm{GT}$, an indicator of bile duct obstruction, has been used to infer hepatocyte damage and the degree of bile duct obstruction (22-24). The results of the present study demonstrated a significant increase in serum levels for all markers of liver function and liver fibrosis, except CHE and PCIII, in the EG.

Du et al (25) observed that, in vitro, in the initial S1 stage of liver tissue fibrosis, the level of PCIII mRNA was increased compared with that of type I collagen; however, with the development of hepatic fibrosis to the S2 stage, type I collagen expression increased markedly. In the present study study, subsequent to pathological grading using hematoxylin and eosin staining, it was observed that the degree of fibrosis in the EG was consistent with the S2 stage, and this was considered to be the primary reason for the lack of a significant alteration in PCIII expression. Increased $\mathrm{CHE}$ levels are associated with chronic, long term hepatic cirrhosis; therefore, the decreased serum levels in the present experimental model were expected.

Serum levels of HA, PCIII, and cIV were all observed to be positively correlated with increased serum levels of the markers of liver function, indicating their potential as indicators of cholestatic hepatic injury.

Histopathological examination has been the primary means of diagnosing hepatic fibrosis and cirrhosis. In the present study, the grading results of hepatic fibrosis in hematoxylin and eosin staining demonstrated that there were significant differences when the EG was compared with the CG or BCG.
Table V. Correlation analysis of liver function test and pathological hepatic fibrosis grade in neonatal cholestatic rats.

\begin{tabular}{lcc}
\hline & \multicolumn{2}{c}{ Statistical analysis } \\
\cline { 2 - 3 } Factor & $\mathrm{r}$ & P-value \\
\hline ALT, U/1 & 0.45 & N.S \\
AST, U/1 & 0.41 & N.S \\
TBIL, $\mu \mathrm{mol} / 1$ & 0.30 & N.S \\
DBIL, $\mu \mathrm{mol} / 1$ & 0.23 & N.S \\
IBIL, $\mu \mathrm{mol} / 1$ & 0.29 & N.S \\
$\gamma$-GT, U/1 & 0.62 & $<0.01$ \\
CHE, KU/l & -0.20 & N.S \\
TBA, $\mu \mathrm{mol} / 1$ & 0.37 & N.S \\
\hline
\end{tabular}

N.S, not significant; ALT, alanine aminotransferase; $\gamma$-GT, $\gamma$-glutamyl transferase; TBIL, total bilirubin; DBIL, direct bilirubin; TBA, total bile acids; CHE, cholinesterase; AST, aspartate aminotransferase; IBIL, indirect bilirubin.

Table VI. Correlation analysis of degrees of hepatic fibrosis and pathological hepatic fibrosis grade in neonatal cholestatic rats.

\begin{tabular}{lcccc}
\hline & \multicolumn{4}{c}{ Factor } \\
\cline { 2 - 5 } $\begin{array}{l}\text { Statistical } \\
\text { analysis }\end{array}$ & $\begin{array}{c}\mathrm{HA}, \\
\mathrm{ng} / \mathrm{ml}\end{array}$ & $\begin{array}{c}\text { PCIII, } \\
\mathrm{ng} / \mathrm{ml}\end{array}$ & $\begin{array}{c}\mathrm{LN}, \\
\mathrm{ng} / \mathrm{ml}\end{array}$ & $\begin{array}{c}\mathrm{cIV}, \\
\mathrm{ng} / \mathrm{ml}\end{array}$ \\
\hline $\mathrm{r}$ & 0.83 & 0.38 & -0.01 & 0.26 \\
P-value & $<0.01$ & N.S & N.S & N.S \\
\hline
\end{tabular}

N.S, not significant; HA, hyaluronic acid; PCIII, procollagen type III; LN, laminin; cIV, collagen type IV. 
The results of the present study demonstrated that the pathological hepatic fibrosis grade was positively correlated with the levels of HA and $\gamma$-GT in serum. The results of the present study additionally indicated that HA and $\gamma$-GT serum levels may serve as sensitive and accurate biomarkers of hepatic fibrosis and degree of injury. The result of the present study may provide a novel, non-invasive means of diagnosis, thus leading to earlier diagnosis and treatment. The results of the present study require validation in preclinical studies prior to being used in human clinical trials, particularly if applied to children and neonates.

\section{Acknowledgements}

The present study was supported by the Key Medical Science Research Program of Hebei Province (grant no. ZL20140195).

\section{References}

1. Zolner G and Trauner M: Mechanisms of cholestasis. Clin Liver Dis 12: 1-26, 2008.

2. Hines JE, Johnson SJ and Burt AD: In vivo responses of macrophages and perisinusoidal cells to cholestatic injury. Am J Pathol 142: 511-518, 1993

3. Zhang YP, Yao XX and Zhao X: Interleukin-1 beta up-regulates tissue inhibitor of matrix metalloproteinase-1 mRNA and phosphorylation of c-jun N-terminal kinase and p38 in hepatic stellate cells. World J Gastroenterol 12: 1392-1396, 2006.

4. Kossor DC, Meunier PC, Handler JA, Sozio RS and Goldstein RS Temporal relationship of changes in hepatobiliary function and morphology in rats following alpha-naphthylisothiocyanate (ANIT) administration. Toxico1 Appl Pharmacol 119: 108-114, 1993.

5. Tang N, Zhang Y, Liu Z, Fu T, Liang Q and Ai X: Correlation analysis between four serum biomarkers of liver fibrosis and liver function in infants with cholestasis. Biomed Rep 5: 107-112, 2016.

6. Gao LN, Yan K, Cui YL, Fan GW and Wang YF: Protective effect of Salvia miltiorrhiza and Carthamus tinctorius extract against lipopolysaccharide-induced liver injury. World J Gastroenterol 21: 9079-9092, 2015

7. Farleigh RM, Knodell RG and Steele NM: Characterization of a hepatic congestion model in the rat: Application of pharmacological studies. Proc Soc Exp Biol Med 166: 134-140, 1981.

8. Li GY, Gao HY, Huang J, Lu J, Gu JK and Wang JH: Hepatoprotective effect of Cichorium intybus L., a traditional Uighur medicine, against carbon tetrachloride-induced hepatic fibrosis in rats. World J Gastroenterol 20: 4753-4760, 2014.

9. Stasi C and Milani S: Non-invasive assessment of liver fibrosis: Between prediction/prevention of outcomes and cost-effectiveness. World J Gastroenterol 22: 1711-1720, 2016.

10. Bedossa P, Dargère D and Paradis V: Sampling variability of liver fibrosis in chronic hepatitis C. Hepatology 38: 1449-1457, 2003 .
11. Cadranel JF, Rufat P and Degos F: Practices of liver biopsy in France: Results of a prospective nationwide survey. For the Group of Epidemiology of the French Association for the Study of the Liver (AFEF). Hepatology 32: 477-481, 2000.

12. Orsler DJ, Ahmed-Choudhury J, Chipman JK, Hammond T and Coleman R: ANIT-induced disruption of biliary function in rat hepatocyte couples. Toxicol Sci 47: 203-210, 1999.

13. Golbar HM, Izawa T, Yano R, Ichikawa C, Sawamoto O, Kuwamura M, Lamarre J and Yamate J: Immunohistochemical characterization of macrophages and myofibroblasts in $\alpha$-Naphthylisothiocyanate (ANIT)-induced bile duct injury and subsequent fibrogenesis in rats. Toxicol Pathol 39: 795-808, 2011.

14. Kim SK and Kim YC: Effects of betaine supplementation on hepatic metabolism of sulfur-containing amino acids in mice. J Hepatol 42: 907-913, 2005.

15. Li SB, Xu FM, Xue C, Ding XJ, Li YC, QIian LY, Zhang GL and Zeng F: Protective effects of ursodeoxycholic acid on a-naphthylisothi-induced acute liver injury in rats. Chin J Digestion 32: 325-329, 2012

16. Lee HH, Seo YS, Um SH, Won NH, Yoo H, Jung ES, Kwon YD, Park S, Keum B, Kim YS, et al: Usefulness of non-invasive markers for predicting significant fibrosis in patients with chronic liver disease. J Korean Med Sci 25: 67-74, 2010.

17. Lijia S, Aifei T and Ruilong X: The clinical value of serum markers of liver fibrosis in patients with viral hepatitis. Jiangxi J Med Lab Sci 25: 453-454, 2007.

18. El-Shabrawi MH, Zein El Abedin MY, Omar N, Kamal NM, Elmakarem SA, Khattab S, El-Sayed HM, El-Hennawy A and Ali AS: Predictive accuracy of serum hyaluronic acid as a non-invasive marker of fibrosis in a cohort of multi-transfused Egyptian children with $\beta$-thalassaemia major. Arab J Gastroenterol 13: 45-48, 2012.

19. Nath NC, Rahman MA, Khan MR, Hasan MS, Bhuiyan TM, Hoque MN, Kabir MM, Raha AK and Jahan B: Serum hyaluronic acid as a predictor of fibrosis in chronic hepatitis $B$ and $C$ virus infection. Mymensingh Med J 20: 614-619, 2011.

20. Nobili V, Alisi A, Torre G, De Vito R, Pietrobattista A, Morino G, De Ville De Goyet J, Bedogni G and Pinzani M: Hyaluronic acid predicts hepatic fibrosis in children with nonalcoholic fatty liver disease. Transl Res 156: 229-234, 2010.

21. Teare JP, Sherman D, Greenfield SM, Simpson J, Bray G, Catterall AP, Murray-Lyon IM, Peters TJ, Williams R and Thompson RP: Comparison of serum procollagen III peptide concentrations and PGA index for assessment of hepatic fibrosis. Lancet 342: 895-898, 1993.

22. Fan L and Zhihua H: Significance of serum 5'-Nucleotidase and $\gamma$-glutamyltranspeptidase in differential diagnosis for infantile hepafitis syndrome and biliary atresia. J Clin Exper Med 8: 16-17, 2009.

23. Tarantino G, Finelli C, Colao A, Capone D, Tarantino M, Grimaldi E, Chianese D, Gioia S, Pasanisi F, Contaldo F, et al: Are hepatic steatosis and carotid intima media thickness associated in obese patients with normal or slightly elevated gamma-glutamyl-transferase? J Transl Med 10: 50, 2012.

24. Tavian D, Degiorgio D, Roncaglia N, Vergani P, Cameroni I, Colombo R and Coviello DA: A new splicing site mutation of the $\mathrm{ABCB} 4$ gene in intrahepatic cholestasis of pregnancy with raised serum gamma-GT. Dig Liver Dis 41: 671-675, 2009.

25. Du W, Zhang Y and Zhai W: A study on type I, II and IV collagen product ion in CCL4 induced rat liver fibrosis. Zhonghua Bing Li Xue Za Zhi 26: 74-77, 1997 (In Chinese). 